\title{
Reviewing code consistency is important, but Research Ethics Committees must also make a judgement on scientific justification, methodological approach and competency of the research team.
}

\author{
Samantha Trace \& Simon E. Kolstoe \\ School of Health Sciences and Social Work, University of Portsmouth
}

We have followed with interest the commentaries arising from Moore and Donnelly's 2015 argument that authorities in charge of research ethics committees (RECs) should focus primarily on establishing codeconsistent reviews [1]. We broadly agree with Savulescu's [2] argument that ethics committees should become more expert, but in a different way and for a different reason.

We have recently been working with the UK Health Research Authority (HRA) analysing the outcomes of their "Shared Ethical Debate" exercises (ShEDs)[3]. Each ShED exercise involves the circulation of a single research project to a number of RECs. The resulting minutes from each REC are compared along with the final decisions made by each REC on the project under consideration. This process was originally an administrative exercise designed to promote greater consistency amongst the 60 or so ethics committees that were brought under the HRA (and previously National Research Ethics Service (NRES)) umbrella within NHS England. Over twenty ShEDs have been run, and although the process has significant weaknesses and has not always been run consistently, the results of the exercises are fascinating. Broadly speaking NHS RECS have been getting more consistent over time in terms of their decision making, but the reasons for the final decisions as described in the committee minutes continue to vary widely. Qualitative research now needs to be done to understand why different committees can have such different discussions in relation to exactly the same research project and yet come to essentially the same conclusion.

As part of our analysis of the ShED data we looked at how RECs across the exercises utilised guidance produced by the HRA, broadly an indication of whether they were being "code consistent". Here it was interesting to notice that the administrator writing the reports for five of the exercises (ShEDs 14, 15, 16, 17 \& 18) stated that RECs made no explicit reference to HRA guidance despite specific guidance on the topics covered by these ShED exercises being available. Conversely only one ShED report (ShED 9) praised the RECs in this regard: "In our analysis we could identify no comments that seemed inappropriate, outside REC's remit or in contravention of guidance." Our analysis indicates that RECs do currently consider code consistency but in a haphazard manner.

One re-occurring issue across all the exercises was the extent to which RECs perform "scientific review" or similarly focused too closely on the scientific methodologies. This is a long running debate within the ethics committee community with some [4] arguing that RECs need to do a much better job of determining the scientific and methodological validity of proposed studies, and yet guidance [5] clearly stating "It is not the task of a REC to undertake additional scientific review, nor is it constituted to do so." Commentaries from researchers indicate considerable irritation when RECs pass judgement based on the scientific or technical aspects of a study[6]. This complaint is perhaps often justified because the broad range of studies reviewed by any particular REC make it unlikely that suitable scientific or clinical expertise will be in the room during the review of a study in the same way as it might be for a grant reviewing committee. However, despite this guidance from authorities and complaints from researchers, "bad science is bad (or at least poor) ethics"[7] because it wastes time, wastes resources, and potentially puts participants at unnecessary risk. Whilst it might be hoped that by the time a study gets to a REC both the scientific justification and methodological validity should be beyond doubt, analysis of REC minutes produced from the ShED exercises, along with the authors experience of sitting on such committees, suggest that this is only occasionally the case. Indeed RECS often find themselves trying to review projects proposed by clinicians with no relevant research experience (for instance researchers sometimes naively arguing "there are no risks, it's only a questionnaire") or students who have received little or no support when developing their protocols. If RECs did not pick up on 
these scientific and/or methodological weaknesses they would be complicit in allowing research waste[4]. RECs are not expert peer review committees, but they do have significant experience in reviewing research protocols, generally know what a good protocol looks like, and according to a 2015 HRA survey, $88 \%$ of researchers find a REC review relevant and useful[8].

RECs are also well placed to assess the researchers themselves and determine whether they have confidence that the research team knows what they are doing and are able to conduct the work appropriately. Again this issue should be beyond doubt prior to research getting as far as an ethics committee, but the ShED exercises have shown that this is often not the case, with RECs frequently commenting on the suitability and sometimes presentation of the research team and their project. A poorly written REC application coupled with researchers who are perhaps dismissive of the review process or unhelpful in their responses to a committee is an ethically problematic issue. Indeed projects are more likely to receive an unfavourable ethics opinion when researchers do not attend ethics committee meetings in person[9]. Research is a complex activity and difficult to do well. It also leads to great rewards that some ambitious researchers and clinicians are keen to obtain without putting in the time required to master the process. Again if RECs did not pick up on this issue no one else in the current research landscape would step in to stop this type of bad research from going ahead.

So what is the role of research ethics committees? Ensuring code consistency is one important function, but another is asking probing questions of researchers in order to make a judgement on the overall package of research being presented. In an ideal world RECs would not have to fulfil this role, but in the current research landscape it seems a necessary and important task. The problem is, as currently constituted within the English NHS system, RECs are not well equipped to evidence their judgements based on peer review by members with the appropriate expertise. Instead the scientific and methodological assessment is carried out by relatively "lay" RECs trying to get a general "feel" for the proposed research and the research team based upon a judgement of competency and professionalism coming from experience of reviewing many similar studies. Sometimes this may be appropriate for catching and stopping research that has not undergone rigorous review prior to reaching the committee, but the current level of experience and training of RECs is not appropriate to do this effectively for all the varied research that is reviewed. We therefore argue that RECs play an important role that extends beyond just monitoring code consistency, but to carry out this additional role they need to be suitably equipped to make assessments of the science, methodology and research team. Perhaps the time has come for professional RECs?

1. Moore A, Donnelly A. The job of 'ethics committees' [Internet]. Journal of Medical Ethics. 2015. Available from: http://jme.bmj.com/content/early/2015/11/13/medethics-2015-102688.short

2. Savulescu J, Moore A, Donnelly A. The structure of ethics review : expert ethics committees and the challenge of voluntary research euthanasia. 2017;2015-8.

3. Trace S, Kolstoe SE. Measuring inconsistency in research ethics committee review. BMC Med Ethics. 2017;18(1):1-10.

4. Chalmers I, Glasziou P. Avoidable waste in the production and reporting of research evidence. Lancet. 2009;374(9683):86-9.

5. NHS. GafREC 2011 [Internet]. 2011. Available from: https://www.gov.uk/government/uploads/system/uploads/attachment_data/file/213753/dh_133993 .pdf

6. Della Sala S, Cubelli R. Entangled in an ethical maze. Psychologist. 2016;29(12):930-2.

7. Hunter D. Bad Science Equals Poor, Not Necessarily Bad, Ethics. Ethics, Law Soc - Vol 3. 2007;61-72.

8. HRA. HRA 2015 Survey [Internet]. 2015. Available from: https://www.hra.nhs.uk/documents/893/assessing-professional-public-opinion-hra-2015.pdf

9. Heasman P, Preshaw P, Gray J. Does researchers' attendance at meetings affect the initial opinions of research ethics committees ? Res Ethics. 2008;4(2):56-8. 